\title{
Osimertinib rechallenge under steroid protection following osimertinib-induced pneumonitis: three case studies
}

Ther Adv Med Oncol

2021, Vol. 13: 1-7

DOI: $10.1177 /$

17588359211018028

(c) The Author(s), 2021. Article reuse guidelines: sagepub.com/journalspermissions

Christiane Bickert*(D, Kathrin Kahnert*, Diego Kauffmann-Guerrero,
Jeremias Götschke(iD, Zulfiya Syunyaeva, Jürgen Behr and Amanda Tufman

\begin{abstract}
Osimertinib is a third-generation tyrosine kinase inhibitor that became the preferred first-line treatment option for metastatic non-small cell lung cancer with sensitizing epidermal growth factor receptor mutations. Drug-induced pneumonitis is known to occur with osimertinib. In case of severe pneumonitis, discontinuation of treatment and therapy with corticosteroids is recommended, and a treatment switch is usually performed. We herein report the treatment course in three patients who were rechallenged with osimertinib under steroid protection following an osimertinib-induced pneumonitis. All our patients were initially re-exposed to a lower dose of osimertinib. Two patients were successfully rechallenged under prednisolone protection. The third patient, who was initially retreated with osimertinib without steroid protection, suffered from a recurrent pneumonitis, and was later rechallenged successfully under steroid protection. Our case series indicates that rechallenge with osimertinib following recovery from osimertinib-induced pneumonitis allows a successful rechallenge in individual cases when alternative treatment options are lacking. Concomitant steroids appear to protect against flares of pneumonitis during rechallenge.
\end{abstract}

Keywords: epithelial growth factor receptor, interstitial lung disease, immunotherapy, nonsmall cell lung cancer, tyrosine kinase inhibitor

Received: 25 January 2021; revised manuscript accepted: 23 April 2021.

\section{Introduction}

Targeted therapies have significantly improved the prognosis of patients with non-small cell lung cancer (NSCLC) harboring activating epidermal growth factor receptor (EGFR) mutations. Osimertinib is a third-generation tyrosine kinase inhibitor (TKI) that has become a very effective first-line treatment option for metastatic NSCLC with sensitizing EGFR mutations. ${ }^{1}$ It demonstrated efficacy against brain lesions, ${ }^{2}$ and improved overall survival compared with patients receiving either gefitinib or erlotinib. ${ }^{3}$ Osimertinib is generally well tolerated, but common side effects include diarrhea, rash, dry skin, and decreased appetite. A rare, but possibly lifethreatening adverse event is pneumonitis, occurring in $1.8-4 \%$ of patients. ${ }^{4,5}$ In case of severe pneumonitis, discontinuation of treatment and therapy with corticosteroids is usually recommended. A treatment switch to other EGFR TKIs or chemotherapy is, especially for patients with brain metastases, problematic due to less efficacy in the central nervous system (CNS) compared with osimertinib. ${ }^{5-7}$ Due to its excellent CNS efficacy and effectiveness in T790Mresistance mutation, ${ }^{5}$ re-exposure to osimertinib would be the preferred treatment option for selected patients, even after severe pneumonitis.

Here, we report the treatment course in three EGFR-positive NSCLC patients who were rechallenged with osimertinib under steroid protection following an osimertinib-induced pneumonitis.
Correspondence to: Christiane Bickert Department of Medicine V, University Hospital, LMU Munich, Member of the German Center for Lung Research, Klinikum Großhadern, Marchioninistr, 15 , Munich, Bavaria 81377. Germany christiane.bickertamed. uni-muenchen.de Kathrin Kahnert Diego KauffmannGuerrero

Jeremias Götschke Zulfiya Syunyaeva Jürgen Behr Amanda Tufman Department of Medicine V, University Hospital, LMU Munich, Member of the German Center for Lung Research, Munich, Bavaria, Germany

*These authors contributed equally to this work. 
Case reports

Patient 1 (58years, female, ex-smoker, 10 packyears) was initially diagnosed with an adenocarcinoma of the left lower-lung lobe (activating EGFR mutation, deletion exon 19) in June 2017. The stage at first diagnosis was cT2aN2M1c (metastatic pattern: diffuse bone metastasis, lymph node metastasis) and treatment with afatinib was initiated. Following a 2-year period of progression-free survival, a follow-up staging showed new brain metastases. Liquid biopsy confirmed the presence of the initial EGFR mutation without T790M-resistance mutation. Off-label treatment with osimertinib ( $80 \mathrm{mg}$ daily) was started due to higher CNS activity. Within 8 weeks, the patient developed dyspnea, and the general condition deteriorated. Lung-function testing showed mild hypoxemia (partial pressure of oxygen $\left[\mathrm{pO}_{2}\right] 71 \mathrm{mmHg}$ ) and a significant decrease of diffusion capacity (TLCO) from $76 \%$ predicted to $55 \%$ predicted. Chest computed tomography (CT) imaging showed bipulmonal ground-glass opacities (Figure 1). There was no evidence of bacterial or viral infection in the bronchoalveolar lavage (BAL), but differential cell count showed a relevant lymphocytosis $(75 \%)$ in the BAL fluid. Based on these findings, osimertinib-induced pneumonitis was diagnosed and intravenous treatment with prednisolone $(1 \mathrm{mg} /$ $\mathrm{kg}$ ) was started and gradually reduced to $5 \mathrm{mg}$ within 4 weeks. After 4 weeks, the patient showed a clinical and respiratory improvement, and rechallenge with osimertinib $40 \mathrm{mg}$ daily under oral steroid protection ( $20 \mathrm{mg}$ prednisolone daily) was started. Within a period of 4 months, prednisolone was gradually reduced (10 mg daily). Suspecting progression of cerebral metastases, we increased the osimertinib dosing (alternating $40 / 80 \mathrm{mg}$ ) under prednisolone protection with $10 \mathrm{mg}$ daily. Diffusing capacity improved to $82 \%$ of predicted 4 months after the beginning of the re-exposition. The dose of osimertinib was increased to $80 \mathrm{mg}$ daily with good tolerability. At the time of this case report, the latest positron emission tomography (PET) CT scan showed no evidence for recurrent pneumonitis and showed stable disease (Figure 1).

Patient 2 (65years, female, never-smoker) was diagnosed with an adenocarcinoma (activating EGFR mutations: ELREA [deletion exon 19],
T790M [exon 20] and L858R [exon 21] mutation) of the left upper-lung lobe. The TNM classification of malignant tumors (TNM) at initial diagnosis was cT3cN2pM1c (metastatic pattern: three CNS lesions; one cerebellar and two occipital cerebral metastases). Stereotactic radiation therapy of the cerebral metastases was performed, and systemic therapy with osimertinib $80 \mathrm{mg}$ daily was initiated. After 7 weeks, the patient presented in the emergency room with increasing respiratory deterioration. Lung function testing showed severe hypoxemia in room air $\left(\mathrm{pO}_{2} 47 \mathrm{mmHg}\right)$ and a significant decrease of the diffusion capacity (TLCO) (34\% predicted compared with baseline of 104\% predicted). The CT scan of the thorax showed basal bipulmonal ground-glass opacities (Figure 2). BAL showed no evidence of acute infection, and a relevant lymphocytosis (58\%) was seen in the BAL differential cell count. Based on these results, pneumonitis was diagnosed. Osimertinib was paused and prednisolone pulse therapy was initiated. The patient experienced quick recovery. At 4 weeks later, progression of the cerebral metastases was observed. Due to the rapid cerebral progress and the patient's reluctance to undergo whole-brain irradiation, re-exposition with osimertinib ( $40 \mathrm{mg}$ daily) under steroid protection $(0.5 \mathrm{mg} / \mathrm{kg}$ prednisolone daily) was started. The patient was monitored closely, and lung-function measurements, including diffusing capacity, were performed weekly. After 2 weeks, follow-up staging showed regression of the primary tumor site, as well as brain metastases. With stable lung function and good tolerability of osimertinib, the dose was increased (alternating $80 \mathrm{mg}$ and $40 \mathrm{mg}$ ) under protection with $30 \mathrm{mg}$ prednisolone daily. The prednisolone dose was reduced to $5 \mathrm{mg}$ daily over the course of the treatment, and the follow-up PET scan showed stable disease 3 months after reexposition (Figure 2). After 5 months of the osimertinib/prednisolone combination, PET CT scan showed recurrent tumor at the primary tumor site, lymph nodes, and brain metastasis. Re-biopsy revealed a new concomitant MET exon 14 skipping mutation and the disappearance of the T790M-resistance mutation. Radiation of the primary tumor was performed, and treatment was completely switched to a combined treatment with afatinib and crizotinib. ${ }^{8}$

Patient 3 (64years, male, never-smoker) was diagnosed with a poorly differentiated adenocarcinoma (activating EGFR mutation, L858R 
(a)

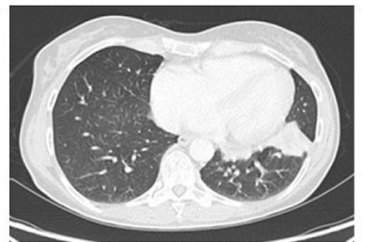

Initial diagnosis 06/2017

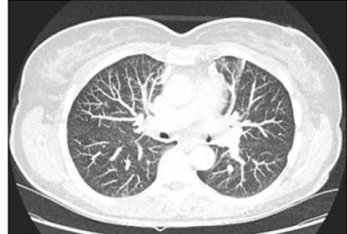

Pneumonitis $10 / 2019$

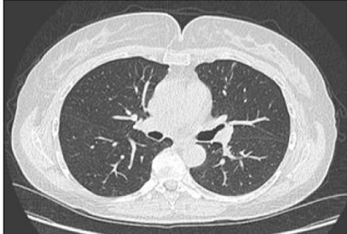

Post pneumonitis $11 / 2019$

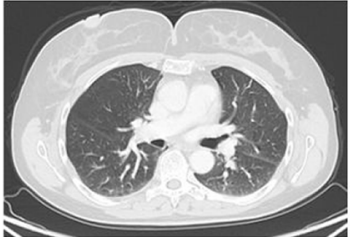

Osimertinib rechallenge $09 / 2020$

(b)

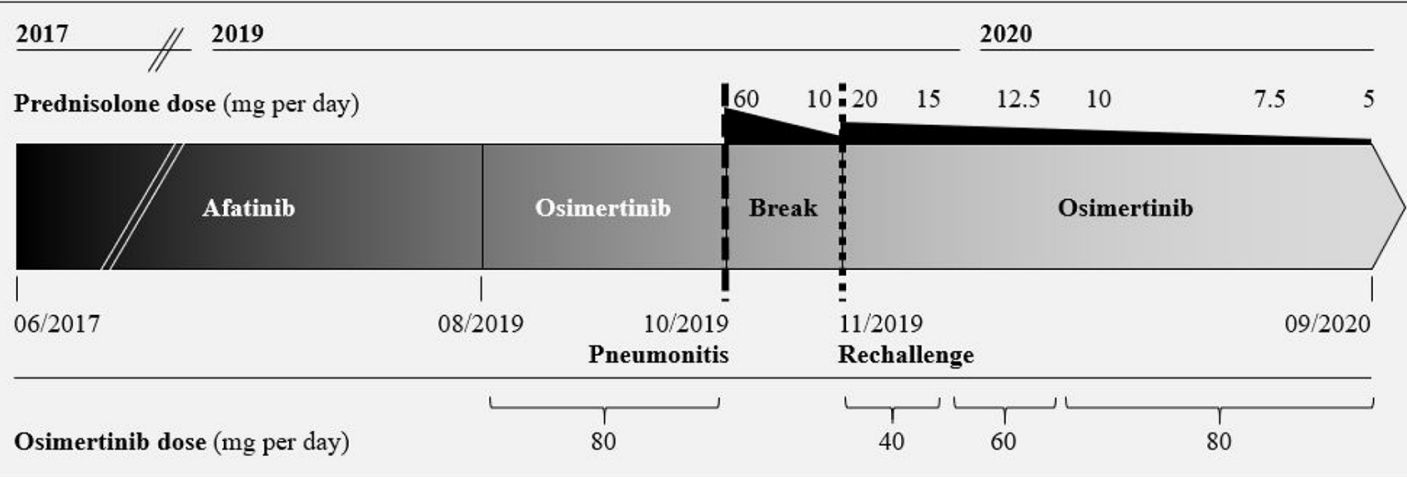

Figure 1. Radiologic and medication details of patient 1.

(a) Radiologic details; (b) details of medication history.

(a)

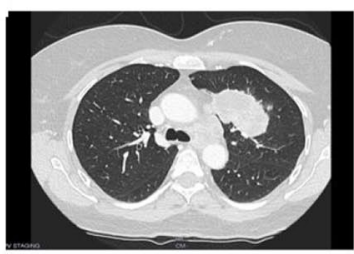

Initial diagnosis $11 / 2019$

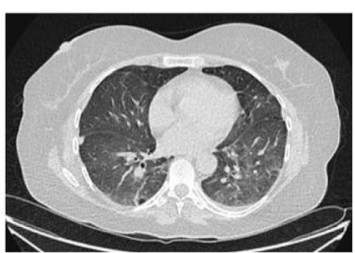

Pneumonitis $01 / 2020$

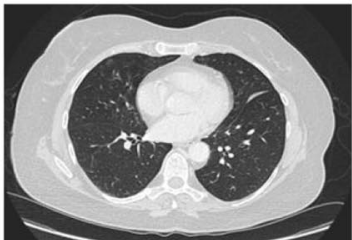

Post pneumonitis $02 / 2020$

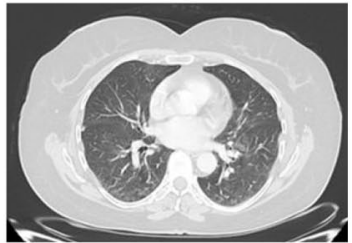

Osimertinib rechallenge $04 / 2020$

(b)

\begin{tabular}{|l|llllllll|}
\hline $\begin{array}{l}\text { Prednisolone dose } \\
\text { (mg per day) }\end{array}$ & 2019 \\
\hline
\end{tabular}

Figure 2. Radiologic and medication details of patient 2.

(a) Radiologic details; (b) details of medication history.

mutation in exon 21) of the right upper-lung lobe. The initial TNM staging was cT3N3M1a. The treatment was initiated with afatinib and later changed to osimertinib due to T790M mutation. After 9 months, the patient suffered from lateonset eosinophilic pneumonitis (grade 3) as 
(a)

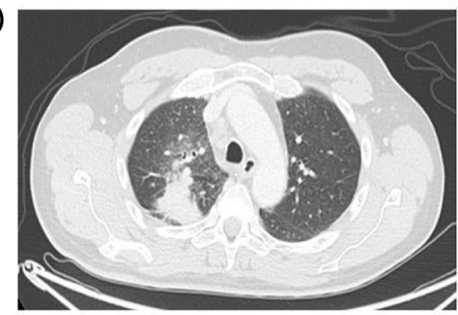

Initial diagnosis $08 / 2016$

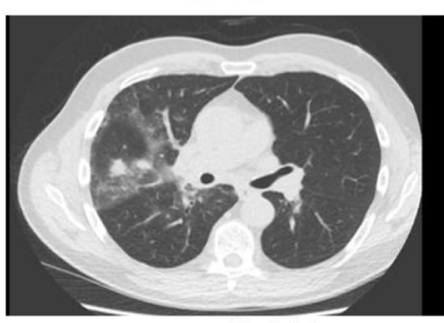

Pneumonitis (II/II) $10 / 2019$

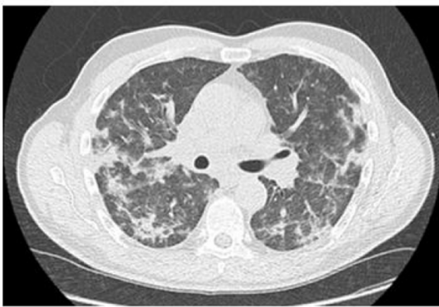

Pneumonitis (I/II) $05 / 2018$

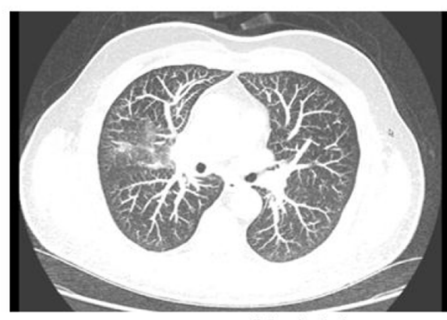

Post pneumonitis (II/II) $11 / 2019$

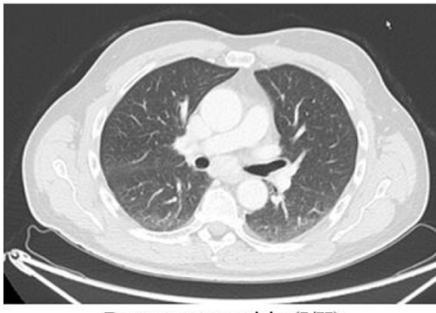

Post pneumonitis (I/II) $06 / 2018$

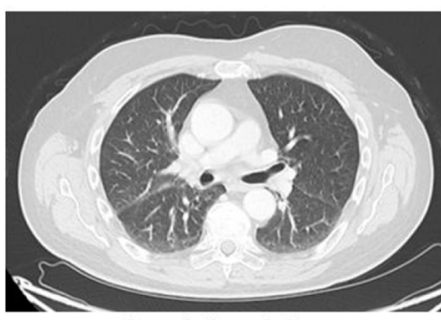

Osimertinib rechallenge $07 / 2020$

(b)

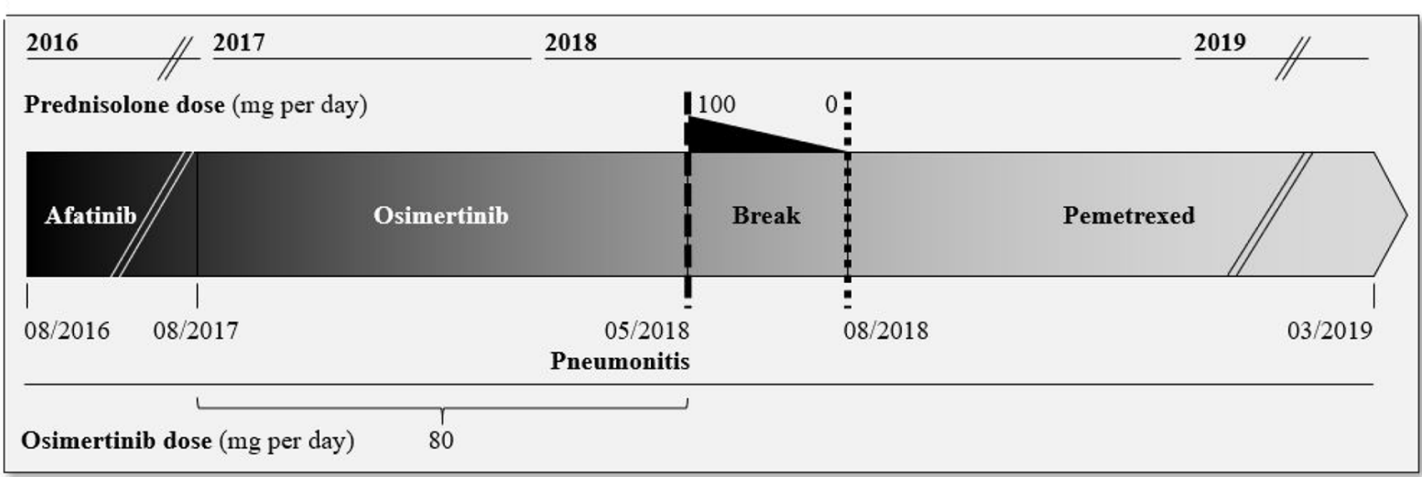

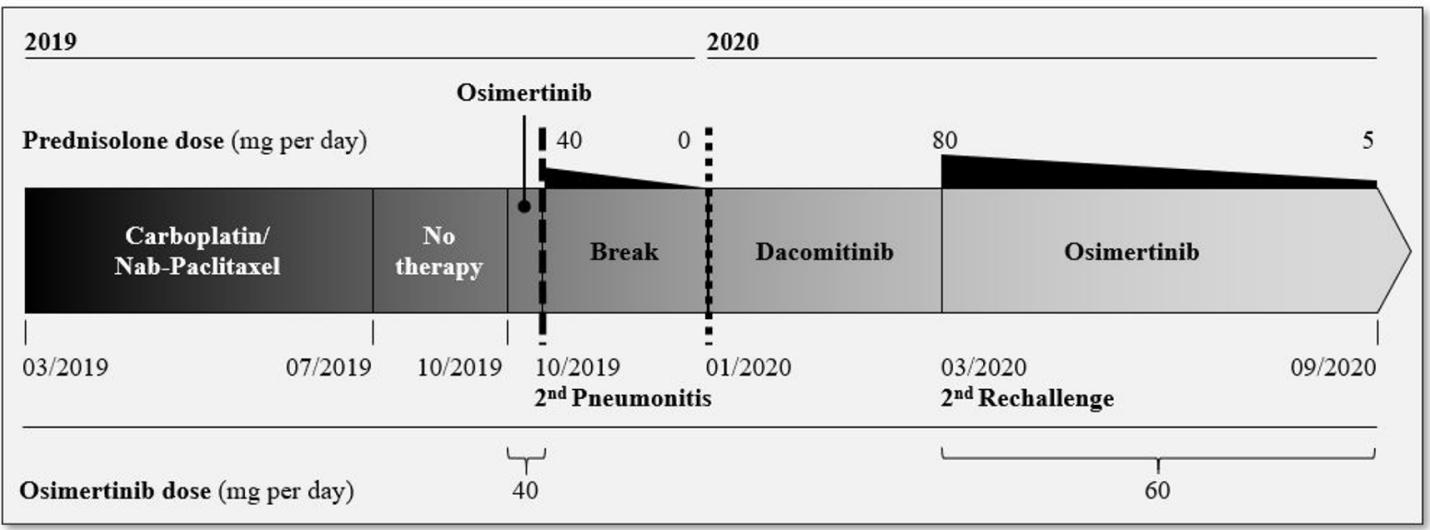

Figure 3. Radiologic and medication details of patient 3. (a) Radiologic details; (b) details of medication history.

described by our research group. ${ }^{9}$ Tumor-specific therapy was stopped for 3 months. Follow-up PET CT scan showed progression of lymphatic metastases, and chemotherapy with pemetrexed was started. Due to a renewed tumor progression, the therapy was switched to carboplatin/nabpaclitaxel. Despite intensified chemotherapy, new brain metastases developed. In consent with the patient, an osimertinib rechallenge ( $40 \mathrm{mg}$ daily, without steroid protection) was performed. 
Within 2 weeks, the diffusion capacity decreased from $90 \%$ to $57 \%$ predicted and the patient suffered from dyspnea. Again, thoracic CT scan showed bipulmonal ground-glass opacities (Figure 3). The differential cell count showed a lymphocytosis (54\%) and $1 \%$ eosinophils in the BAL fluid. Recurrent pneumonitis was suspected, and prednisolone pulse therapy led to rapid clinical improvement. Tumor-specific treatment was switched to dacomitinib. Within 12 weeks, the tumor showed further progression with multiple new lung metastases, as well as progression of brain metastases. Despite the high risk of reoccurrence of potential life-threatening pneumonitis, due to missing alternative treatment options the patient agreed to renewed treatment with osimertinib. Osimertinib was started ( $40 \mathrm{mg}$ daily) with accompanying prednisolone therapy $(1 \mathrm{mg} /$ $\mathrm{kg}$ body weight), which was gradually decreased to $40 \mathrm{mg}$ prednisolone daily within 3 weeks. With good clinical tolerability and stable lung function, osimertinib dosage was increased to alternating $40 / 80 \mathrm{mg}$ daily and prednisolone was gradually reduced to $5 \mathrm{mg}$ daily. At 6 months after the second osimertinib rechallenge, the patient is in excellent clinical and respiratory condition and follow-up PET CT scans, as well as brain magnetic resonance imaging, showed stable tumor status.

\section{Discussion}

This case series shows that re-exposure to osimertinib is possible despite previous drug-induced pneumonitis. All three patients received concomitant prednisolone, which may have prevented pneumonitis flares. Osimertinib is an efficient third-generation TKI and is a preferred first-line therapy for EGFR-mutated NSCLC. ${ }^{10}$ Previous investigations showed an advantage of osimertinib compared with the combination of platinum and pemetrexed therapy ${ }^{5}$ and to standard EGFR-TKIs. ${ }^{4}$ Particularly, the effect of osimertinib on CNS is superior to other TKIs. ${ }^{11}$ CNS penetration of osimertinib is $16 \%{ }^{12}$ and therefore higher than CNS penetration rate of erlotinib (2.5-13\%), ${ }^{13-15}$ gefitinib (1.1$3.58 \%),{ }^{14,16-18}$ and afatinib (under 1\%). ${ }^{19}$ The pivotal study of dacomitinib excluded patients with CNS metastases, and the CNS penetration rate has not been evaluated yet. ${ }^{20}$ In addition, osimertinib is so far the only medication that adequately treats the resistance mutation T790M. This is the reason why in daily clinical practice there is often a need to re-administer osimertinib despite previous serious side effects.
A rare, but life-threatening adverse event of osimertinib is pneumonitis, with mortality rates of $10-25 \%{ }^{4,5,21}$ In case of pneumonitis, discontinuation of osimertinib treatment and therapy with corticosteroids is recommended. Further treatment options are chemotherapeutic treatment approaches, or alternative TKIs. However, erlotinib, gefitinib, afatinib and dacomitinib may only have little success in treating brain lesions due to limited CNS penetration. ${ }^{13-19}$

Considering the risks and benefits of alternative treatment options, osimertinib retreatment should be discussed. Rechallenge after osimertinib-induced pneumonitis was reported in eight patients during the past 3 years. ${ }^{22-28}$ The mechanism of osimertinib-induced pneumonitis is not fully understood, but in association with other drug-induced interstitial lung diseases, the pathogenesis is multifactorial, including a significant part of immunoreaction such as release of cytocines. ${ }^{29}$ This indicates that steroids could potentially protect from recurrent pneumonitis in case of rechallenge. To confirm the diagnosis of pneumonitis we recommend, whenever possible, a bronchoscopy with BAL to exclude infectious causes and to prove lymphocytosis in the BAL.

We believe that osimertinib rechallenge can be a safe therapeutic option for patients under close clinical monitoring. All our patients were initially re-exposed to the lower dose of $40 \mathrm{mg}$ daily. Two patients were successfully rechallenged under prednisolone protection $(0.5 \mathrm{mg} / \mathrm{kg}$ body weight $)$. The third patient was initially re-exposed to osimertinib without steroid protection, suffered from a recurrent pneumonitis and was later rechallenged successfully under steroid protection. This underlines that patients benefit from steroid protection, and that it should be considered when rechallenging a patient with osimertinib.

Our case series is in line with previous results, indicating that osimertinib rechallenge under steroid protection is an appropriate treatment option for patients. We showed that steroid protection allows a successful rechallenge in individual cases when alternative treatment options are lacking. Nonetheless, future research is needed to evaluate the lowest effective prednisolone dosage, the respective reduction scheme, and the starting dose, as well as the gradual enhancement of osimertinib to offer an effective and safe osimertinib rechallenge in EGFR-mutated patients. 


\section{Conclusion}

This small case series describes osimertinib rechallenge as an appropriate treatment option for patients with advanced EGFR-mutated NSCLC despite previous osimertinib-induced pneumonitis. Furthermore, our report highlights the relevance of concomitant treatment with steroids. The optimal steroid dosage should be evaluated in further studies.

\section{Conflict of interest statement}

Dr Bickert, Dr Kahnert, Dr Götschke, Dr Syunyaeva and Dr Behr: none.

Dr Kauffmann-Guerrero reports personal fees from AstraZeneca, personal fees from Boehringer Ingelheim, personal fees from Pfizer, grants and personal fees from Takeda, outside the submitted work.

Dr Tufman reports personal fees from Lilly, grants and personal fees from AstraZeneca, personal fees from Takeda, personal fees from Roche, personal fees from Celgene, personal fees from MSD, personal fees from BMS, personal fees from Pfizer, outside the submitted work.

\section{Funding}

The authors received no financial support for the research, authorship, and/or publication of this article.

\section{Ethics and patients}

Written informed consent was given by the patients for the publication of the cases and figures. A copy of the signed consent form is available on request. Approval was obtained from the Institutional Ethics Committee (project number 21-0057 KB).

\section{ORCID iDs}

Christiane Bickert (iD https://orcid.org/00000003-4121-6687

Jeremias Götschke (iD) https://orcid.org/00000002-3426-2194

\section{References}

1. Planchard D, Popat S, Kerr K, et al. Metastatic non-small cell lung cancer: ESMO clinical practice guidelines for diagnosis, treatment and follow-up. Ann Oncol 2018; 29: iv192-iv237.

2. Ballard P, Yates JWT, Yang Z, et al. Preclinical comparison of osimertinib with other EGFR-

TKIs in EGFR-mutant NSCLC brain metastases models, and early evidence of clinical brain metastases activity. Clin Cancer Res 2016; 22: 5130-5140.

3. Ramalingam SS, Vansteenkiste J, Planchard $\mathrm{D}$, et al. Overall survival with osimertinib in untreated, EGFR-mutated advanced NSCLC. $N$ Engl f Med 2020; 382: 41-50.

4. Soria J-C, Ohe Y, Vansteenkiste J, et al. Osimertinib in untreated EGFR-mutated advanced non-small-cell lung cancer. $N$ Engl $\mathcal{F}$ Med 2018; 378: 113-125.

5. Mok TS, Wu Y-L, Ahn M-J, et al. Osimertinib or platinum-pemetrexed in EGFR T790M-positive lung cancer. N Engl f Med 2017; 376: 629-640.

6. Reungwetwattana T, Nakagawa K, Cho BC, et al. CNS response to osimertinib versus standard epidermal growth factor receptor tyrosine kinase inhibitors in patients with untreated EGFRmutated advanced non-small-cell lung cancer. FCO 2018; 36: 3290-3297.

7. Barlesi F, Gervais R, Lena H, et al. Pemetrexed and cisplatin as first-line chemotherapy for advanced non-small-cell lung cancer (NSCLC) with asymptomatic inoperable brain metastases: a multicenter phase II trial (GFPC 07-01). Ann Oncol 2011; 22: 2466-2470.

8. Kauffmann-Guerrero D, Kahnert K, Kumbrink $\mathrm{J}$, et al. Successful treatment of a patient with NSCLC harboring an EGFR mutation and a concomitant met exon 14 skipping mutation combining afatinib and crizotinib. Clin Lung Cancer 2019; 20: 59-62.

9. Syunyaeva Z, Berghof K, Kauffmann-Guerrero $\mathrm{D}$, et al. Late-onset severe pneumonitis under osimertinib. AME Case Rep 2019; 3: 39.

10. National Comprehensive Cancer Network clinical practice guidelines in oncology (NCCN guidelines). Non-Small Cell Lung Cancer. Version 8. 2020.

11. Heon S, Yeap BY, Lindeman NI, et al. The impact of initial gefitinib or erlotinib versus chemotherapy on central nervous system progression in advanced non-small cell lung cancer with EGFR mutations. Clin Cancer Res 2012; 18: 4406-4414.

12. Yang JCH, Kim S-W, Kim D-W, et al. Osimertinib in patients with epidermal growth factor receptor mutation-positive non-small-cell lung cancer and leptomeningeal metastases: the BLOOM study. FCO 2020; 38: 538-547.

13. Togashi Y, Masago K, Hamatani Y, et al. Successful erlotinib rechallenge for leptomeningeal metastases of lung adenocarcinoma after erlotinib-induced 
interstitial lung disease: a case report and review of the literature. Lung Cancer 2012; 77: 464-468.

14. Togashi Y, Masago K, Masuda S, et al. Cerebrospinal fluid concentration of gefitinib and erlotinib in patients with non-small cell lung cancer. Cancer Chemother Pharmacol 2012; 70: 399-405.

15. Deng Y, Feng W, Wu J, et al. The concentration of erlotinib in the cerebrospinal fluid of patients with brain metastasis from non-small-cell lung cancer. Mol Clin Oncol 2014; 2: 116-120.

16. Zhao J, Chen M, Zhong W, et al. Cerebrospinal fluid concentrations of gefitinib in patients with lung adenocarcinoma. Clin Lung Cancer 2013; 14: 188-193.

17. Chen Y, Wang M, Zhong W, et al. Pharmacokinetic and pharmacodynamic study of gefitinib in a mouse model of non-small-cell lung carcinoma with brain metastasis. Lung Cancer 2013; 82: 313-318.

18. Franceschi E, Cavallo G, Lonardi S, et al. Gefitinib in patients with progressive highgrade gliomas: a multicentre phase II study by Gruppo Italiano Cooperativo di Neuro-Oncologia (GICNO). Br F Cancer 2007; 96: 1047-1051.

19. Hoffknecht P, Tufman A, Wehler T, et al. Efficacy of the irreversible ErbB family blocker afatinib in epidermal growth factor receptor (EGFR) tyrosine kinase inhibitor (TKI)pretreated non-small-cell lung cancer patients with brain metastases or leptomeningeal disease. F Thorac Oncol 2015; 10: 156-163.

20. Wu Y-L, Cheng Y, Zhou X, et al. Dacomitinib versus gefitinib as first-line treatment for patients with EGFR-mutation-positive non-small-cell lung cancer (ARCHER 1050): a randomised, open-label, phase 3 trial. Lancet Oncol 2017; 18: 1454-1466.
21. Ahn M, Tsai C, Shepherd FA, et al. Osimertinib in patients with T790M mutation-positive, advanced non-small cell lung cancer: long-term follow-up from a pooled analysis of 2 phase 2 studies. Cancer 2019; 125: 892-901.

22. Miyauchi E, Ichinose M and Inoue A. Successful osimertinib rechallenge in a patient with T790M-mutant non-small cell lung cancer after osimertinib-induced interstitial lung disease. $\mathcal{F}$ Thorac Oncol 2017; 12: e59-e61.

23. Mamesaya N, Kenmotsu H, Katsumata M, et al. Osimertinib-induced interstitial lung disease after treatment with anti-PD1 antibody. Invest New Drugs 2017; 35: 105-107.

24. Kiriu T, Tamura D, Tachihara M, et al. Successful osimertinib rechallenge with steroid therapy after osimertinib-induced interstitial lung disease. Intern Med 2018; 57: 91-95.

25. Nagasaka M and Gadgeel SM. Retreatment with osimertinib following pneumonitis. Clin Lung Cancer 2018; 19: e53-e55.

26. Satoh S, Shiroyama T, Tamiya M, et al. Successful osimertinib rechallenge after osimertinib-induced pneumonitis in a patient with lung adenocarcinoma. Respir Med Case Rep 2018; 23: 68-70.

27. Lu H and Dowell J. Osimertinib in pulmonary manifestations: two case reports and review of the literature. In Vivo 2020; 34: 315-319.

28. Ahn JH. Successful osimertinib retreatment after extremely early onset severe pneumonitis in firstline treatment of lung adenocarcinoma. Thorac Cancer 2020; 11: 2713-2716.

29. Camus P, Fanton A, Bonniaud P, et al. Interstitial lung disease induced by drugs and radiation. Respiration 2004; 71: 301-326.
Visit SAGE journals online journals.sagepub.com/ home/tam

@SAGE journals 